\title{
Intelligent Facility Management for Sustainability and Risk Management
}

\author{
Adam Kučera and Tomáš Pitner \\ Masaryk University, Faculty of Informatics \\ Laboratory of Software Architectures and Information Systems \\ Botanická 68a, 60200 Brno, Czech Republic \\ akucera@mail.muni.cz, tomp@fi.muni.cz
}

\begin{abstract}
Building construction has gone through substantial change with the emerging spread of ICT during last decades. In the field of construction industry, the term intelligent buildings describes facilities equipped with devices and systems that can be remotely controlled and programmed and that are able to communicate and collaborate in order to ensure convenient building environment and effective operation. However, installing devices with such capabilities is only one part of the task of effective facility management and risk management. Facility managers have to be provided with tools that allow them to inspect and analyze gathered building operational data and make decisions to improve building performance. Decision support tools for facility managers usually lack deep integration with building systems. This article aims on technical aspects of providing data from intelligent buildings to the responsible personnel in order to help them to operate the building effectively with emphasis on its energy consumption and risk management.
\end{abstract}

Keywords: computer-aided facility management, energy management, building management systems, intelligent buildings, business intelligence, monitoring systems, sustainable development, risk management.

\section{Introduction}

Facility management as a term emerged in the 1970's. International Facility Management Association defines facility management as a profession that encompasses multiple disciplines to ensure functionality of the built environment by integrating people, place, process and technology.

The technology part of the facility management have experienced great change as information and communication technologies (ICT) are widely used in various building systems such as building automation, security systems or decision support software. In the construction business, the term intelligent building is used mostly for facilities equipped with remotely controlled and monitored interoperating devices. Other definitions of the term are discussed in [1].

In the opposition to use of the ICT on the low level of ensuring the technical part of building operation such as air conditioning, heating or security in

J. Hřebíček et al. (Eds.): ISESS 2013, IFIP AICT 413, pp. 608-617, 2013.

(C) IFIP International Federation for Information Processing 2013 
real time and constantly, ICT helps facility managers to analyze and evaluate the building performance in a long-term perspective using business intelligence and decision support tools, namely CAFM (Computer-Aided Facility Management) software products. CAFM software covers wide range of activities that are not part of organization's core business, but are necessary for its operation space management, hoteling and reservations, preventive and on demand maintenance, service desk, portfolio management, and accounting. As the main goal of CAFM products is to lower the expenses spent on every day operation, energy management became a regular part of CAFM software products.

Reliable data about organization's facilities (device location, building plans, documentation) can be also used for preparation for the critical sitations (natural disasters, technologcial failures,... ) or for direct decision support during disasters and thus increase safety of building users and reduce recovery costs.

By intelligent facility management, we mean utilizing above mentioned ICT technologies (namely capabilities of intelligent building systems and facility management software) to make facility operation inexpensive and more efficient, energy undemanding and thus more sustainable and resistant to failures or disasters. This article proposes selected improvements and extension to the building systems concerning the reliability, accessibility of the data, and cooperation with other systems that do not participate on the operation directly, namely CAFM software and other information systems used in the organization.

\section{Integration Technology in Facilities}

A wide variety of electronic systems is usually installed in modern buildings - e.g. $H V A C$ (heating, ventilation, air condition) regulation and automation (closely connected with environmental protection), security system, access control system, CCTV (Closed-Circuit Television), fire alarm system, lighting control. Integration of all the systems that take part on the building operation is mostly motivated by economical reasons. The ability to control remotely and to monitor all systems in the building from a common user interface reduces the number of staff needed for ensuring the operation as well as demands on expert knowledge of the system operators. A common interface for all the systems also allows them to cooperate (e.g. an air conditioning unit in a particular room cannot be switched on when a window is open - in this use case, the security system provides data from the magnetic sensor on the window frame and passes them to the air conditioning control unit). Rules similar to this one improve the work environment in the buildings and save energy. In this way, purely economical considerations and actions help to make the building operation more sustainable. A building that is able to efficiently decrease its energy consumption is cheaper and more environment-friendly.

The integration of building systems is usually accomplished by establishing a common communication bus that uses one of the integration protocols. Hardware and software vendors support various protocols that can be used on a common bus, e.g. BACnet, LONWorks or KNX/EIB. The bus interconnects operators' workstations and application servers (web user interface, archive database 
servers, monitoring systems) with the back-end devices such as programmable logic controllers (PLCs), central units of security systems or with protocol gateways and translators. The described infrastructure (workstations, servers, gateways, common bus) is referred to as a Building Management System (BMS).

\subsection{Building Management at the University Campus Bohunice}

The project of the Building Management System that spreads over the whole Masaryk University (BMS MU) and its facilities started together with the construction of the first part of the University Campus in Brno-Bohunice (UKB) that was completed in 2007. Since then, the UKB site has grown to 30 buildings controlled by a common BMS. Additionally, other buildings spread through the City of Brno and belonging to various organization units (Institute of Computer Science, Faculty of Economics, University Rectorate/Headquarters, Accommodation and Catering Services) were connected to the system at the occasions of replacing obsolete building equipment with new units. Other projects under construction are planned to be connected to the BMS MU in the next few years. See Figure 1 for schema of the BMS UKB infrastructure.

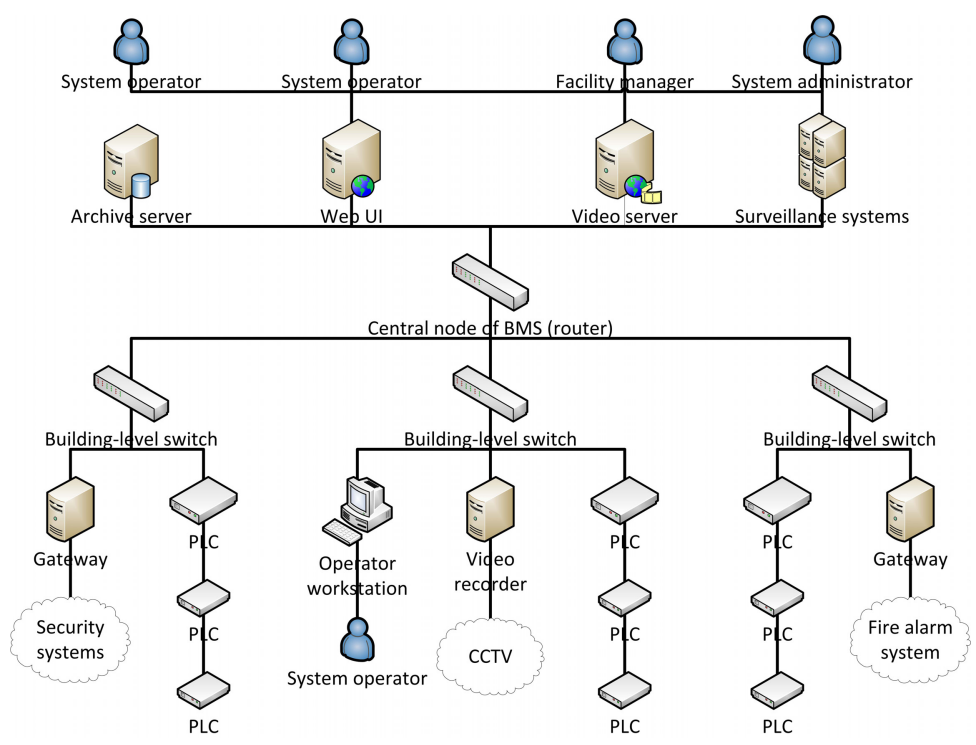

Fig. 1. BMS infrastructure schema (Source: Authors)

The systems integrated in BMS of MU vary on different phases of construction of UKB and other sites. The following systems are integrated under the BMS interface on most of the buildings (bold systems have environmental impact): 
1. HVAC

2. Lighting control

3. Elevator monitoring

4. Energy monitoring

5. Access control system

6. Security system

7. Fire alarm system

8. CCTV (Closed-Circuit Television)

9. UPS monitoring

The core of the system is formed by PLCs and application servers that communicate over BACnet protocol standardized by ISO 16484-5. BMS MU today contains over 700 BACnet-capable devices from more 10 vendors including Delta Controls, Saia-Burgess, Honeywell, Johnson Controls, Siemens, and WAGO.

The users are provided with a web interface that allows them to control and monitor all integrated systems. The system contains two archive servers for saving historical data of the building operation.

\section{Requirements for an Effective Facility Management}

Equipping a building with advanced technologies with the ability to communicate and collaborate on the building operation does not guarantee efficient use of the resources or environment-friendly behavior of the facility. To make the building intelligent in such a way that installed systems are actually helping to optimize expenses and energy consumption of the organization facilities or help to react to the crises (natural disasters, failures), additional tools are required according to our experience gathered during five years of the UKB operation. There is a need for advanced monitoring and evaluation of building performance emerging especially at relatively large sites (dozens of buildings) such as the UKB. Currently available state-of-the-art CAFM systems support analyses based on indirect indicators such as energy consumption (electricity, water, gas) based on data gathered from the invoices from energy suppliers. These techniques usually do not provide enough detail to detect the source of poor operation effectiveness.

Further parts of the paper introduce advanced tools and methods for building performance analysis and evaluation with respect to environmental impact and risk management based on the data gathered directly from the Building Management System devices. These data provide us with a direct insight into the processes and relations among various systems incorporated in building operation and thus allows us to perform a detailed drill-down in order to inspect and resolve possible malfunctions, breakdowns, problems or errors emerging in the systems.

\section{Monitoring}

During the operation of relatively large sites of the UKB, the need for specialized monitoring tools for the BMS emerged. With increasing number of devices 
connected to the BMS, constant surveillance of all parts of the system by human operators becomes impossible. Since devices involved in the building operation usually do not support standard methods for network management such as SNMP and ICMP protocols, existing tools must either be adapted for the BMS environment or new tools must be developed. At the UKB, both approaches have been tested and used in the production environment.

An IP traffic flow-based network monitoring tool was extended by a BACnet stack for the analysis of the BACnet traffic, see [2]. A monitoring device (probe) acts as a passive listener on central nodes of the BMS network and monitors most of the network traffic occurring in the network by inspection of Ethernet frames. The data from the probe can be used for detection of sources of suspicious, atypical communication which usually comes from misconfigured devices. An incorrectly communicating device represents serious threat to the stability of the whole network as misconfigured devices tend to use broadcast messages extensively. An early detection of such devices saves time of the building operators which can be spent on more productive tasks such as optimization of building performance and system cooperation which lead to efficient operation.

A specialized monitoring tool for BACnet (named BMSSentinel) network is developed at Masaryk University for the purposes of permanent accessibility and functionality surveillance of key elements in the BMS network such as application servers, protocol gateways, specific PLCs ensuring precise climate conditions in labs, or routing devices. BMSSentinel utilizes SCADAEngine BACnet Server $A P I 1$ for accessing the BACnet network and defines variety of custom algorithms for detecting specific types of faults occurring in the BMS network. BMSSentinel provides users with web user interface and automatic notifications about detected problems. The tool is similar to the Nagios infrastructure monitoring tool well known from the field of ordinary ICT. Since Nagios software is used at Masaryk University, BMSSentinel is able to pass detected problems to the Nagios server where they can be processed the same way as other alerts coming from computer network.

Capabilities of BMSSentinel ensure instant reactions to network accidents, errors or failures. Besides that, the system focuses on ensuring data validity and completeness. Data correctness is a fundamental requirement for further analysis of building performance which can lead to operation optimization. Real-time monitoring of system function provides decision support in the case of various failures happening in the system and helps system operators to detect the root cause and correspondingly react to it. An overview of currently used monitoring and traffic analysis tools is provided in Table 1.

\section{New Control Mechanisms for the BMS}

Building management systems usually base their control decisions (switching devices on and off, opening and closing valves,...) on the data from following inputs:

\footnotetext{
${ }^{1}$ http://scadaengine.com/
} 
Table 1. Monitoring tools, protocols and functionality (Source: Authors)

\begin{tabular}{|c|c|c|c|c|}
\hline Tool Device & Application server & Gateway & PLC & Switch/router \\
\hline BMS Sentinel & Functionality & Functionality, Accesibility & Functionality, Accesibility & \\
\hline Network traffic probe & $\begin{array}{l}\text { Communication } \\
\text { characteristics }\end{array}$ & $\begin{array}{l}\text { Communication } \\
\text { characteristics }\end{array}$ & $\begin{array}{l}\text { Communication } \\
\text { characteristics }\end{array}$ & \\
\hline Nagios & Accesibility & Accessibility & & Functionality, Accessibility \\
\hline & \multicolumn{4}{|c|}{ Is: } \\
\hline
\end{tabular}

1. Static regulation algorithms

2. Human operator commands

3. Preset values (requested temperature in the room)

4. Attached sensors (temperature sensors, humidity sensors, pressure sensors, magnetic sensors,...)

Other data sources should be considered, because the effectivity of the BMS is influenced by variety of other factors that cannot be obtained from the above sources.

Other information systems used in the organization often contain data that could be used for more precise setting of the regulation algorithms. The most significant examples of such data sources are the room reservation system and the lecture rooms timetables in case of an educational organization. Time intervals of the reservations can be used as inputs for the algorithm that automatically changes state of centrally controlled air conditioning units. A basic approach with relatively static schedules set by human operators usually reflect different settings for workdays and weekends or holidays and does not reflect dynamic changes in room utilization during the day. The further step to adjust performance of the HVAC systems according to the actual needs requires additional information about the number of occupants in the room in the given time intervals.

Using weather information is another way of improving HVAC performance. The characteristics and amount of the weather data varies from simple usage of outside temperature (which can easily be measured by standard BMS devices) to utilization of other data such as the intensity of solar radiation which must be obtained by specialized devices.

An extensive overview of various approaches to HVAC systems optimization is provided in [3] and [4].

\section{Business Intelligence Tools}

A BMS deployed at a large site is able to generate huge amount of data. In case of the UKB, the archive server stores approximately 100000 new records per day. To obtain useful information about complex processes and events occurring during the building operation, advanced methods of data processing and analysis 
must be applied [5]. Tools provided by BMS vendors (reporting capabilities, data export tools) are very ineffective (user unfriendly with insufficient speed and limited management options) when dealing with large systems.

Complexity and size of the system makes the task of maintaining constant awareness of the state of all parts of the system difficult. However, overview of all the important processes and states occurring in the whole system during crisis is crucial to succesful risk management.

This section introduces several solutions and methods used at the UKB that simplifies the analysis of building behavior. Figure 2 provides an overview of system connections proposed further.

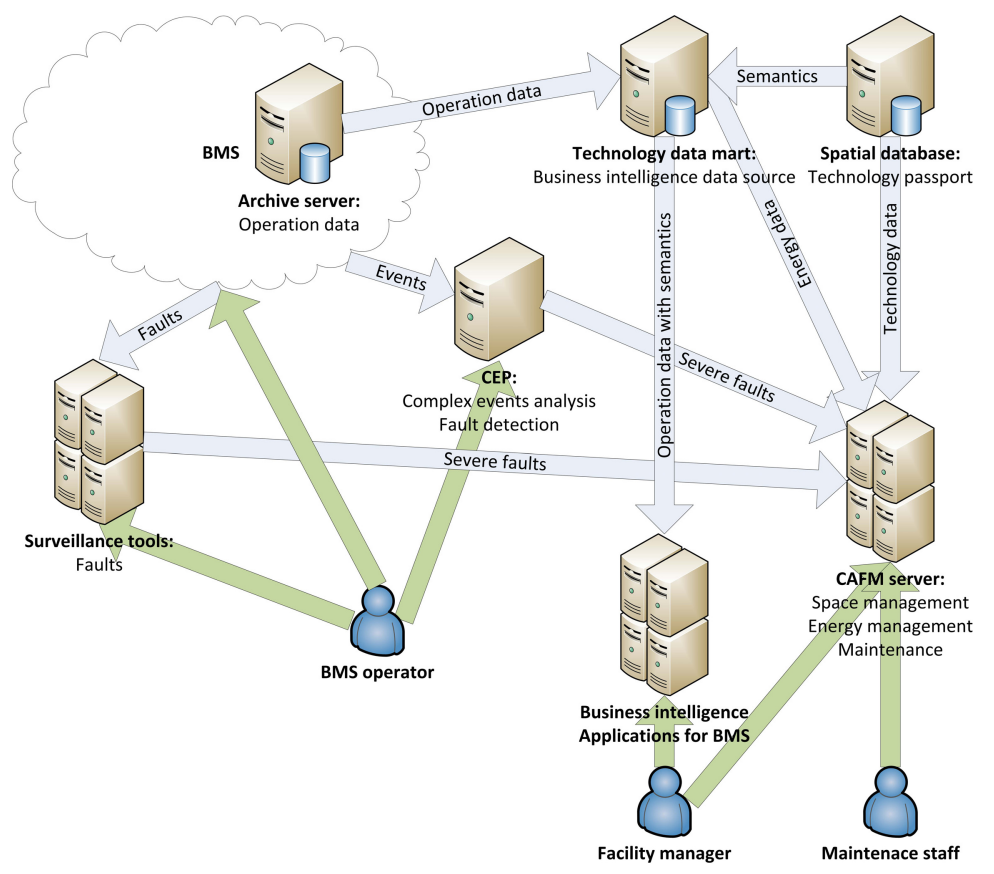

Fig. 2. BMS for Business intelligence use (Source: Authors)

\subsection{Adding Semantics - Integration with Geographic Information Systems}

Data points (values that are monitored using BMS) do not carry any kind of semantic information about their meaning. They are identified using their network addresses for the purpose of the BMS itself. They have names which are designed to be easily understandable by human operators and thus not suitable for machine processing.

In order to perform advanced data analyses such as aggregations of values based on particular features of data points (e.g. average of all room temperatures in specific building), we need additional source of information about the value 
represented by a particular data point. For the purposes of Masaryk University, spatial database of the technology passport can be employed. The technology passport carries information about devices installed in buildings of Masaryk University. Along with its geographical position, each device has a set of attributes attached - the most important for the purpose of integration with the BMS is the type of device. By extending the relation between a data point in BMS and a device in technology passport by a measured or controlled quantity, the data point gains complete semantic information about its precise location, its source device (temperature sensor, humidity sensor, energy meter,...) of the value and the measured or controlled quantity (room temperature, requested room temperature, relative air humidity, current, voltage,...). The relation of data points and devices in the technology passport can be utilized in business intelligence and decision support tools that are described in the following text.

\subsection{Technology Data Mart}

Database schema of BMS archive server has several features that limit its use for business intelligence application. Besides missing semantic information, the archive server suffers from low performance when retrieving results of complex queries that work with long time periods. This is caused by optimization of database structure (both schema and physical) for write operations. In this way, archive server is typical example of OLTP database. For this reasons, implementation of separate data warehouse (Technology data mart - TDM) was tested and is planned for deployment into the operation environment.

TDM (implemented using Microsoft SQL Server 2012) contains information about relations between BMS and technology passport as well as optimized indexing schemas, history tracking and advanced features for operations with time interval.

\subsection{Complex Event Processing}

Complex Event Processing (CEP) is set of tools and techniques allowing us to handle events from large systems in real time. CEP in commonly used in network security, algorithmic stock-trading, fraud detection or business process monitoring for correlating, aggregating and analyzing various events.

For the use at UKB, existing CEP framework (Microsoft StreamInsight) was adapted to support operations based on data point semantics and is able to provide building operators with easily understandable outputs that help to optimize building operation and detect various types of faults. A prototype of the system was implemented and tested in real operation environment. The system is able to perform selections, filtering and computations (average, summa, maximum, minimum) based on the data point semantic information. System runs continuous user-defined queries that are evaluated in sliding time windows. Results of the computations performed by the system can by further analyzed automatically (e.g. detecting faults of a particular device by comparing other devices on the same type) or stored for later visualization that can be examined by users. 


\subsection{Machine Learning and Data Mining}

The data from the BMS can be examined by methods of machine learning (ML) and data mining. Algorithms as decision trees, outlier detection or clustering can be used to discover complex rules that occur in the building behavior. ML analysis, however, suffers from unavailability of data that prevents the algorithms to provide useful results. Many factors that influence the building behavior are not measurable by the BMS (the number of occupants in the room), not measured because of technological or economical limitations (a limited number of sensors that can be attached to the device) or are not saved into the archive server due to the performance reasons.

Despite this limitations, the use of ML and data mining from building management data is a promising research topic. Different approaches to analysis based on ML approaches are presented in [6], 7] and [8].

\section{Risk and Crisis Management}

The University Campus of Masaryk University is not prone to specific kinds of natural disasters and crises such as floods or earthquakes thanks to its location on the hill in geologically stable area of Central Europe. The main threats concerning natural influences are risk of fire and massive downfalls. However, events such as electricity blackouts or extensive system failures require immediate and corresponding reactions to current situation development.

For such situations, BMS in conjunction with CAFM software are able to provide managers and operators with precise, credible, relevant and up-to-date information. Real-time data about system statuses enriched by semantics obtained from spatial databases can be visualized using the facility management data (building plans) and provide overall view current situation that helps responsible personnel to make correct decisions in the time of crisis, disaster or failure. Cooperation of systems integrated in the BMS allows the facility to automatically react to the situations comprising health and/or safety risks and minimize the impacts of the crisis without the human intervention (i.e. fire safety system is able to control HVAC devices in order to prevent the fire from spreading caused by running ventilation).

\section{Conclusion}

This paper presents the field of facility management of intelligent buildings and proposes technical requirements for ICT parts of intelligent building systems that must be fulfilled in order to ensure a cost effective, energy efficient and sustainable building operation that is not prone to crises such as system failures or natural disasters. The requirements are based on experience gathered during the first 5 years of operation of the Masaryk University campus in Brno-Bohunice. Next, we propose methods to satisfy defined requirements. Large sites require a complex approach to data analysis and fault detection. Some of the presented 
solutions are used in the production environment while some of them are planned for future development. The building management data brings a significant potential to improve the building performance in the means of energy efficiency as well as management of risks and crises, but mining useful information from large amounts of data is not a trivial task and suitable methods for data processing must be applied.

Acknowledgement. This work was supported by the European Unions territorial cooperation program European Territorial Co-Operation Austria-Czech Republic 2007-2013 of the under the EFRE grant M00171, project iCom (Constructive International Communication in the Context of ICT).

\section{References}

1. Wong, J.K.W., Li, H., Wang, S.W.: Intelligent building research: a review. Automation in Construction 14(1), 143-159 (2005)

2. Krejčí, R., Čeleda, P., Dobrovolný, J.: Traffic Measurement and Analysis of Building Automation and Control Networks. In: Sadre, R., Novotný, J., Čeleda, P., Waldburger, M., Stiller, B. (eds.) AIMS 2012. LNCS, vol. 7279, pp. 62-73. Springer, Heidelberg (2012)

3. Prívara, S., Široký, J., Ferkl, L., Cigler, J.: Model predictive control of a building heating system: The first experience. Energy and Buildings 43(2-3), 564-572 (2011)

4. Cigler, J., Prívara, S., Váňa, Z., Žáčeková, E., Ferkl, L.: Optimization of Predicted Mean Vote index within Model Predictive Control framework: Computationally tractable solution. Energy and Buildings 52, 39-49 (2012)

5. Kriksciuniene, D., Pitner, T., Kucera, A., Sakalauskas, V.: Sensor Network Analytics for Intelligent Facility Management. In: Frontiers in Artificial Intelligence and Applications. Intelligent Interactive Multimedia Systems and Services, vol. 254, pp. 212-221. IOS Press (2013)

6. Popelínský, L., Glos, P.: Towards Detection of Anomalies in Building Management Data. In: 15th IBIMA conference on Knowledge Management and Innovation: A Business Competitive Edge Perspective, pp. 664-669. IBIMA Publishing (2010)

7. Seem, J.E.: Pattern recognition algorithm for determining days of the week with similar energy consumption profiles. Energy and Buildings 37(2), 127-139 (2005)

8. Seem, J.E.: Using intelligent data analysis to detect abnormal energy consumption in buildings. Energy and Buildings 39(1), 52-58 (2007)

9. Shen, W., Hao, Q., Xue, Y.: A loosely coupled system integration approach for decision support in facility management and maintenance. In: Automation in Construction, vol. 25, pp. 41-48. Elsevier B.V., Amsterdam (2012) 